Instituto Internacional de Investigación y Desarrollo Tecnológico Educativo INDTEC, C.A.

DOI: https://doi.org/10.29394/Scientific.issn.2542-2987.2018.3.8.10.197-217

OAI-PMH: http://www.indteca.com/ojs/index.php/Revista Scientific/oai

\title{
La Participación Ciudadana como vía para abordar la acción Transformadora de la Escuela Primaria
}

\author{
Autora: Sonia María Peña Guzmán \\ Universidad Latinoamericana y Del Caribe, ULAC \\ profesorasonia12@gmail.com \\ Caracas, Venezuela
}

\section{Resumen}

La transformación de la Escuela Primaria requiere de espacios donde se brinde una educación constructivista, participativa y popular, en la que se origine el pensamiento crítico, reflexivo y participativo de los estudiantes, en el que el docente cumpla el rol de facilitador y promotor de la participación, así como de la participación de los estudiantes, padres, madres y representantes. Es por ello, que la Participación Ciudadana permite el fortalecimiento de las capacidades de las personas, grupos y organizaciones. Sin embargo, en la Escuela Básica Estadal Dolores González, se evidenció bajos niveles de participación de padres, madres y representantes en el proceso de enseñanza y demás actividades pedagógicas. El objetivo general se basó en generar una teoría centrada en la participación ciudadana como vía para abordar la acción transformadora de la integración Escuela-Comunidad. El enfoque epistemológico del estudio se ubicó en la fenomenología y como método se utilizó el fenomenológico, las técnicas usadas fueron la entrevista a profundidad y la observación. Los informantes claves comprendieron cuatro docentes y cuatro padres y representantes. Como hallazgos fundamentales se aprecia que la participación ciudadana desde la relación escuela-comunidad es escasa, limitándose únicamente a las acciones administrativas del plantel, a saber, asistencia a reuniones, retiro de boletas informativas. Como recomendaciones se sugiere, la implicación de la familia para beneficiar la convivencia y un clima socioafectivo favorable para el libre desarrollo integral de los estudiantes.

Palabras clave: nacionalidad; acción comunitaria; escuela. 


\title{
Citizen Participation as a way to approach the Transforming action of the Primary School
}

\begin{abstract}
The transformation of the Primary School requires spaces where a constructivist, participative and popular education is offered, in which the critical, reflective and participative thinking of the students originates, in which the teacher fulfills the role of facilitator and promoter of the participation, as well as the participation of students, parents, and representatives. That is why the Citizen Participation allows the strengthening of the capacities of individuals, groups and organizations. However, at the Dolores González State Basic School, low levels of participation of parents, mothers and representatives in the teaching process and other pedagogical activities were evidenced. The general objective was based on generating a theory centered on citizen participation as a way to approach the transformative action of the SchoolCommunity integration. The epistemological approach of the study was located in the phenomenology and as a method the phenomenological was used, the techniques used were the in-depth interview and the observation. The key informants included four teachers and four parents and representatives. As fundamental findings it is appreciated that citizen participation from the schoolcommunity relationship is scarce, limited only to the administrative actions of the school, namely attendance at meetings, withdrawal of information slips. As recommendations are suggested, the involvement of the family to benefit the coexistence and a favorable socio-affective climate for the free integral development of the students.
\end{abstract}

Keywords: nationality; community action; school.

Date Received: 26-09-2017

Date Acceptance: 29-11-2017 


\section{Introducción}

La participación es hoy un tema de gran interés para las organizaciones sociales, las instituciones públicas, los centros educativos y las universidades. Por lo tanto, se constituye así en un espacio común de interés entre varios agentes sociales. En la actualidad tiene vigencia en el contexto sociopolítico la participación social y ciudadana en los distintos procesos de la vida venezolana, para ello el Estado enmarca los principios de una democracia participativa y protagónica. Mosonyi (2007:272), afirma: "Ahora bien, si la clave del progreso está en la participación autónoma colectiva, es necesario que desde ahora, y de forma inmediata, las comunidades y asociaciones voluntarias comiencen su proceso de autogestión, echando mano a todos los medios disponibles".

Así mismo, Rey y Santamaría (2010): plantean que Colaborar significa trabajar con otras personas en una misma tarea. Participar es tener parte en algo. Desde esta perspectiva, colaborar es implicarse en la tarea, es tener un proyecto común y ocuparse de él, es un deber más que un derecho y se relaciona con la misión de centro. La participación es, ante todo un derecho constitucional, y está relacionada fundamentalmente con la organización, la estructura y el control.

Ahora bien, las escuelas, están haciendo esfuerzos por generar un proceso de calidad educativa, asumen como parte de su práctica diaria los aspectos que consideran positivos para el logro de la transformación de la escuela, tomando para ello la participación de todos los entes que conforman el proceso educativo, llámense personal directivo, docentes, estudiantes, padres, madres, representantes, denominados entonces como ciudadanía partícipe del objeto educativo. Es necesario destacar que, respecto a la ciudadanía, (Borja, 2002-2003:176): se refiere al "conjunto de individuos de un Estado, con acceso al cúmulo de derechos y deberes políticos que cada uno de ellos tienen".

Con respecto a la acción transformadora le corresponde a la escuela no 
solamente educar al niño, sino extender su acción a todos los entes que la integran: padres, madres, representantes y comunidad en general; buscando vías que hagan más efectiva la incorporación de todos ellos para que se vinculen a la sociedad en una forma dinámica, responsable y creativa.

La relación entre el Estado, la escuela y los docentes constituye la esencia de la doctrina del Estado Docente, y esta a su vez el soporte filosófico, político y social del currículo. La Educación Primaria es el segundo nivel del sistema educativo venezolano, su finalidad es la de contribuir con la formación integral de los niños, niñas y adolescentes, mediante el desarrollo de su potencial creativo y el pleno ejercicio de su personalidad. Currículo Nacional Bolivariano: Diseño curricular del sistema educativo bolivariano (2007).

Siguiendo con el Diseño Curricular, es de aseverar, que la visión de la escuela es lograr la formación integral de cada ser humano en un conjunto de valores tales como, el conocimiento pleno de las raíces y el acervo histórico como pueblo. Formación en, por y para la democracia a través de la participación, consciente y solidaria a través de los procesos de transformación social y de gestión de los asuntos públicos, respetuoso de las leyes y de la pluralidad cultural y étnica. Formación en, por y para el trabajo, corresponsable en su proceso de formación, así como el desarrollo de su capacidad investigativa e innovadora en saber hacer científico, humanístico, técnico, tecnológico, artístico, al servicio de la sociedad, mediante un currículo flexible y contextualizado

Es por ello, que toca a todos los actores educativos la tarea de profundizar en los cambios curriculares que se quieren, hacerlo práctico, coherente, en correspondencia con las necesidades del país y adecuado a la realidad. Se parte de una evaluación minuciosa de la situación escolar para crear la escuela a partir de la participación protagónica y corresponsable de todos. Como parte de las acciones encaminadas a fortalecer la labor escolar mediante el proceso de evaluación, con el firme propósito de mejorar el logro 
educativo y la transformación del currículo, se considera la auto evaluación como punto de referencia para orientar la reflexión y efectuar el diagnóstico de la situación escolar.

De acuerdo con los razonamientos anteriores que se han venido realizando, en la sociedad venezolana surge la necesidad de la participación ciudadana y de su transformación, como en cualquier otra sociedad cambiante y compleja, de manera pues que las escuelas están haciendo esfuerzo por generar un proceso de cambios.

En el caso del Estado Bolivariano de Miranda y las escuelas adscritas al Ejecutivo Regional son las que más se benefician de este proceso de transformación educativa que busca la calidad y excelencia, por cuanto se nutre de los lineamientos generales emitidos por el Ministerio del Poder Popular para la Educación en vía de un diseño curricular Bolivariano pero también de la política que orienta la Dirección General de Educación del Ejecutivo Regional en los cuales la participación de los padres, madres y representantes es la base para que se desarrollen los mismos.

Toda esta conjunción de lineamientos de ambos entes educativos, hacen que las escuelas estadales desarrollen diversas actividades en pro de la transformación educativa, esto se evidencia en los programas regionales: Programa Integral de lectura y alfabetización social (Pilas), Anímate con las Matemáticas (ANIMATE), Promotores de Paz, Mi Programa de Alimentación Escolar, (MIPAE), Escuela de Directores de Escuela, Escuelas para Padres y los Programas Nacionales. Manos a la Siembra, Programa Canaima, Sociedades Bolivarianas, Consejos Escolares, Colección Bicentenaria. Todos ellos insertos en el PEIC (proyecto educativo integral comunitario) y a su vez en los proyectos pedagógicos de aula (PPA).

En la institución educativa de Educación Primaria "Dolores González" adscrita a la Región Educativa Independencia del Ejecutivo Regional del Estado Bolivariano de Miranda, a través de conversaciones informales y observación 
directa en trabajo de campo, se evidencia en esta bajos niveles de participación de padres, madres y representantes en el proceso de enseñanza y demás actividades pedagógicas realizadas dentro de la escuela a través de los programas antes mencionados, es decir, poco se evidencia ese compromiso a través de responsabilidades asumidas con el docente y el estudiante al compartir actividades de enseñanza aprendizaje y de evaluación.

Es de aseverar que los padres, madres y representantes, no aportan ideas relacionadas con aspectos que se toman en cuenta en la evaluación del escolar y en la planificación del docente, tales como asistir a talleres para padres, recibir información para la elaboración de asignaciones en el hogar, realizar trabajos en conjunto con los niños dentro del aula, colaborar con la ejecución de trabajos prácticos con el escolar, entre otros referidos a los antes mencionados.

En este mismo orden y dirección, en la planificación, ejecución y evaluación del proyecto pedagógico de aula tampoco se evidencia ese compromiso de responsabilidades asumidas con el docente y el estudiante al compartir actividades de apoyo de parte del representante, relacionadas con las antes mencionadas.

Aunado a esto, se observa la ausencia de la comunidad en las actividades culturales, actos, mantenimiento de áreas comunes, reparación de ambientes, recursos, muebles, entre otros que benefician a su representado.

De la misma manera, a través de la observación directa de la investigadora durante las actividades cotidianas se observa: apatía, desmotivación, desinterés generalizado por parte de los docentes, personal obrero y administrativo para realizar sus desempeños y afrontar los problemas cotidianos.

Se observa en ellos el desamor al trabajo, la falta de sentido de pertenencia y de estrategias para incentivar la participación ciudadana del representante y la comunidad en general, basando su labor solo en la enseñanza dentro de las aulas con sus alumnos, sin querer participar en nada 
más.

Por todas estas razones, se evidencia que la escuela no se acerca y posesiona de su comunidad y no se observa un centro de formación permanente para el cambio cultural, pese a los grandes esfuerzos que vienen realizando los entes gubernamentales antes descritos.

Cabe decir, que no se observa un docente comprometido con su desempeño, que sirva de modelo de trabajador eficiente, que planifique actividades que contengan rutinas de trabajo que acerquen a la comunidad y enriquecerse de ella, al mismo tiempo no le brindan los insumos para concebir el perfil del ciudadano que se desea, pues se observa un docente centrado solamente en la instrucción y no en la creación, el redescubrimiento y aplicación de conocimientos que permita la solución de problemas.

En función de lo antes señalado, el estudio que se desarrolló se planteó como objetivo: Generar una teoría centrada en la participación ciudadana como vía para abordar la acción transformadora de la escuela, con la articulación de los elementos que coadyuvan a la calidad educativa, específicamente en las instituciones educativas de Educación Primaria adscritas a la Región Educativa Independencia del Ejecutivo Regional del Estado Bolivariano de Miranda.

Del contexto anterior surgen las siguientes interrogantes:

¿Cuál es el significado qué le atribuyen los docentes a la participación ciudadana para el logro de la acción transformadora?.

¿Qué elementos intervienen en la participación ciudadana en el contexto de la Escuela Primaria desde la perspectiva de los padres y representantes?.

¿Cómo es el proceso de la acción transformadora que se efectúa en la integración escuela-comunidad?

¿Cuáles son las categorías emergentes de la participación ciudadana 
para abordar la acción transformadora de la integración escuela comunidad?.

¿Cuáles son los elementos que conforman una teoría centrada en la participación ciudadana para abordar la acción transformadora de la integración escuela - comunidad en las instituciones adscritas a la Región Educativa Independencia del Ejecutivo Regional del Estado Bolivariano de Miranda?

\section{Marco Referencial.}

\subsection{Participación Ciudadana}

La Participación ciudadana es un proceso mediante el cual los habitantes de una nación tienen el derecho a intervenir individual 0 colectivamente, en las diversas acciones comunes para la toma de decisiones sobre los asuntos públicos que afecten en lo político, económico y social.

Asimismo, la participación ciudadana es el proceso que integra al ciudadano en la toma de decisiones, fiscalización, control y ejecución de las acciones en los asuntos públicos y privados, para permitirle su pleno desarrollo como ser humano y el de la comunidad en la cual se desenvuelve.

En este sentido Pérez y Salazar (2009), señala que:

Es Participación Ciudadana la imprescindible participación de todos los hombres y mujeres que quieran implicarse en los problemas que les afectan, aportando puntos de vista, inquietudes y soluciones. La Participación Ciudadana es el acto de, siendo un ciudadano, intervenir en la vida pública y privada del país. (pág. 101).

De acuerdo con lo antes citado, se entiende que la participación ciudadana; estando sujeta al proceso de transformación que es logrado a través de respuestas estratégicas que impulsan a compensar las necesidades y demandas de la nación, valorando la integridad de la comunidad como gestora en la acción comunitaria con el fin de prestar una formación más 
productiva.

Ahora bien, los objetivos explícitos de la participación ciudadana en la Administración Pública pueden organizarse desde tres áreas: la económica, la política y la administrativa. Es así como se detallan a continuación: (Fermín, 2003).

En el área económica: El principal objetivo es la posibilidad de que los ciudadanos y la sociedad organizada puedan direccionar el gasto público, controlar la calidad de inversión y la eficiencia del uso.

En el área política: El principal objetivo es la democratización del sistema político, es decir, la ampliación de los sujetos políticos al conjunto de ciudadanos, comunidades y organizaciones sociales de diferente tipo. Se requiere apuntar que la democratización del sistema político comienza de abajo hacia arriba con la puesta en marcha en el ámbito nacional de los Consejos Locales de Planificación Pública (CLPP) y los Consejos Estadales de Planificación y Coordinación de Políticas Públicas (CEPCPP). Estas serán las primeras instancias de participación y decisión en los asuntos públicos del Estado en la dimensión territorial municipal.

En el área administrativa: La transparencia y la difusión masiva de los procesos de toma de decisiones. La moralización de la administración pública a través del control social y la observación permanente de los ciudadanos.

Con base a lo anterior, se sostiene que la participación ciudadana en los asuntos económicos, administrativos y políticos es parte de la creación de una nueva cultura de compromiso directo de los ciudadanos, las comunidades y la sociedad organizada con el Estado, los gerentes públicos y las políticas sociales de interés colectivo, donde se le brinde solución a los problemas confrontados por la comunidad.

Bajo este contexto, todos los actores deben involucrarse para la acción transformadora no solo de las comunidades a las que pertenece sino en cada una de las instituciones inmersas en dichas comunidades, tal es la escuela 
que conforma un elemento fundamental para la participación.

\subsection{Acción Transformadora de la Escuela Primaria}

La transformación educativa con criterios de calidad y pertinencia social, demanda generar que todos sus actores sean realmente beneficiados y puedan incorporarse eficazmente al sector socio productivo, por cuanto está basada en la pertinencia social, vinculada con la línea estratégica del Proyecto Nacional Simón Bolívar, Primer Plan Socialista - PPS (2007-2013), donde requiere crearse precedentes para un nuevo modelo socio-productivo-político e ideológico, incluyente y garante de la formación del nuevo ciudadano.

Asimismo, requiere concretarse una transformación social, donde es fundamental la transformación del modelo educativo, por ello, la sinergia de las instituciones educativa es determinante para alcanzar una oferta académica con pertinencia social, calidad, equidad, incluyente, para formar ciudadanos con ética, profesionalismo y criterio humanista.

Es así, que las instituciones educativas del país requieren orientar su enfoque hacia la participación ciudadana y aportar con base a la realización de proyectos sociales que beneficien la escuela y el contexto que la rodea, abordados desde la relación comunidad-escuela desde la participación, como posibilidad de construir o desarrollar el conocimiento tanto tácito como explícito a partir del dialogo de saberes.

Todo lo antes expresado, a modo de elemento emancipador, dando resultado el conocimiento que se enriquece con la incorporación de la diversidad de visiones y cosmovisión de todos y cada uno de los involucrados en ese proceso, docentes, estudiantes, comunidad, logrando de esta manera consolidar la macro estructura que se requiere en pro de consolidar el progreso del conocimiento, saberes y aprendizajes en los planteles educativos.

En consecuencia, al tener la educación un papel relevante como agente dinamizador y fortalecedor de los procesos transformadores en la sociedad, el 
sistema educativo formal cumple un rol esencial en la gestión de la participación ciudadana particularmente en la construcción de conocimientos y en el desarrollo de habilidades, actitudes y valores que integran lo social con sus interacciones, entre ellos, la solidaridad, la justicia social, la cooperación, la equidad, el trabajo en equipo, la tolerancia, el respeto por la vida y la diversidad, el actuar, entre otros.

Obviamente, la educación actual requiere ser una educación constructivista, participativa y popular al servicio de las necesidades de la sociedad en donde se promueva el pensamiento crítico, reflexivo y participativo de los estudiantes para así producir una educación multidireccional, en donde el docente cumpla el rol de facilitador y promotor de la participación, así como de la integración de todos los estudiantes con la realidad social que vive la escuela y la comunidad donde habitan.

Ahora bien, bajo tales premisas no es posible entender la construcción de conocimiento transformador en otra perspectiva que no sea la de sujetos activos en la indagación de su realidad. Por lo tanto, en la educación dialógica los estudiantes tienden a reflexionar sobre su propia situación y son parte activa de todo el proceso educativo.

Se puede argumentar que, en la transformación de la escuela primaria, el estudiante requiere ser actor principal de su propio desarrollo, actuando de manera autónoma en su aprendizaje y en la autorrealización de su ser en el futuro.

De esta manera el docente debe mantener esta visión y actuar de forma tal que este escolar pueda tener las bases apropiadas para alcanzar este fin, este docente con visión de futuro debe entrelazar la participación ciudadana al introducir dentro de su práctica además de nuevas estrategias la inclusión de los padres en este desarrollo.

Además, se puede agregar que la educación viene avanzando en una política sintetizada en Educación de Calidad para todos, lo cual implica 
fortalecer la atención educativa al estudiante, aumentando la cobertura y expectativa de vida escolar por un lado, por otro, entrar en un proceso de transformación curricular que implique transitar hacia una educación emancipadora y dignificadora en el marco de los principios constitucionales.

\section{Contexto Metodológico.}

\subsection{Enfoque Epistemológico aplicado a la Investigación}

Desde el enfoque epistemológico, el estudio se fundamenta en la fenomenología, por cuanto se parte de la premisa de que cada grupo humano establece una dinámica de funcionamiento y de relación de una manera particular, así como es específica su forma de relacionarse con otros grupos humanos. Como primer exponente, la escuela filosófica de Husserl, para quien la tarea de la filosofía fenomenológica es constituir a la filosofía como una ciencia rigurosa. Además, hace énfasis en lo individual y sobre la experiencia subjetiva.

Por su parte, Rusque (2003a), señala que la fenomenología:

Se trata de un método original, donde el interés por la investigación se sitúa en el ámbito de la elaboración conceptual. La dinámica va de la realidad concreta a la construcción de la misma, permitiendo que la inteligibilidad haga posible explicitar su forma lógica a través de conceptos (pág. 22).

Bajo este contexto, el estudio analizó la formulación de elementos teóricos de la participación ciudadana desde una visión de la dinámica innovadora en la transformación de la escuela primaria contextualizado en los docentes, padres, madres y representantes de las Escuelas de Educación Primaria adscritas a la Región Educativa Independencia del Ejecutivo Regional del Estado Bolivariano de Miranda.

Así mismo, considerando que desde el mirar de la fenomenología se logró descubrir y describir las esencias y relaciones esenciales existentes en la realidad, 
específicamente en las instituciones educativas, donde se pudieron establecer las diferentes relaciones educativas, sociales, de convivencia, de participación y acción académica.

\subsection{Postura Metodológica}

La metodología del estudio se enmarcó en el paradigma cualitativo, ya que el mismo permitió definir la realidad como es vivida y percibida en la Escuela Estadal Dolores González, destacando las ideas, experiencias, sentimientos y motivaciones de los docentes, padres, madres y representantes de dicha institución educativa.

Esta investigación implicó realizar revisión teórica y el trabajo de campo a través de las acciones en la praxis educativa y social, para abarcar el fenómeno objeto de estudio, se analizó lo que sucede en el espacio y tiempo, con el fin de hallar las aplicaciones usuales y habituales de los actores.

Ahora bien, para la ejecución del trabajo de campo se establecieron los primeros contactos con los informantes clave, que de acuerdo con Martínez (2006a): sugiere que, en el campo educativo los posibles grupos a estudiar serian: los directivos, docentes, padres, representantes y los estudiantes de las instituciones. Cada uno de estos grupos dependiendo del tópico que se desea esclarecer, ilustraría una faceta de la misma realidad.

En tal sentido, se hizo posible la selección de las personas entrevistadas, escogiendo como informantes o fuentes de información a cuatro (4) docentes y cuatro (4) padres, madres y representantes de la Escuela Estadal Dolores González, Municipio Independencia del Estado Bolivariano de Miranda, adscrita a la Región Educativa Independencia del Ejecutivo Regional del Estado Bolivariano de Miranda.

Posteriormente para el trabajo de campo de elaboró un cronograma de las actividades a cumplir para realizar dicho trabajo, luego se seleccionó las técnicas para la recolección de la información, usando la entrevista a 
profundidad y la observación participante; después se hizo contacto con los informantes para el establecimiento de fecha para la aplicación de la entrevista; seguidamente a través de la organización de la información, se procesó, chequeó, organizó y limpio la información recogida.

\subsection{Validez y Fiabilidad de la Investigación}

Toda investigación con paradigma cualitativo requiere tener criterios de validez y confiabilidad, ya que los mismos conducen a la verdad. Para establecer la confiabilidad se asumió el criterio de Rusque (2003b): en el cual propone que el investigador debe respetar ciertas reglas que permiten aumentar la fiabilidad interna, los cuales son:

- Relatos textuales condensados: son producciones verbales de los sujetos, palabra por palabra.

- El diario del investigador donde se consignan las reflexiones, ideas, dudas y confesiones del trabajo en el terreno. Este aspecto está referido a las notas de campo tomadas por el investigador y las cuales están resumidas en las redes semánticas realizadas para establecer la síntesis conceptual.

- Consignación de análisis e interpretación provisoria.

\section{Técnicas de Análisis de la Información}

El procedimiento para el análisis de la información se realizó tomando en cuenta las fases señaladas por Rusque (2010c), las cuales son:

1.- Representación: A través de la presentación de gráficas explicativas, la cual permitirá explicar los datos descritos.

2.- Condensación de los Datos, a través de la interpretación de los mismos. Exige una reflexión sistemática y crítica de todo el proceso de investigación.

El análisis se apoyó en la triangulación, expuesta por Martínez (2006b): 
"lo que permitió contrastar los datos obtenidos con la opinión de los investigadores", es decir, se realizó una comparación de información desde tres aspectos diferentes. De acuerdo con la Universidad Pedagógica Experimental Libertador (2002), la triangulación: “... es una de las técnicas de análisis de datos más característicos de la metodología cualitativa. El principio básico consiste en recoger y analizar datos desde distintos ángulos para contrastarlos entre sí". (pág. 120).

Para este estudio se realizó una triangulación metodológica, donde se establecieron las coincidencias y divergencias entre las informaciones obtenidas de los docentes, padres, madres y representantes.

Por tal motivo, el análisis de la realidad fue un proceso continuo, interpretándola en el desarrollo del estudio, de tal forma, que toda la información recabada, permitió ver la coherencia, coincidencia, incongruencias entre lo que manifestaron los entrevistados.

Finalmente, se teorizó por medio del análisis e interpretación del contexto investigado. Martínez (2006c): establece que para alcanzar este proceso no se sigue una receta o una serie de pasos establecidos; pues la teoría es una construcción simbólica, verbal o icónica, es un modelo ideal el cual ofrece una estructura conceptual inteligible, sistemática y coherente para ordenar los fenómenos, y a una teoría se llega a través de una investigación creativa (pág. 87).

\section{Generación de Teoría}

\subsection{Propósitos}

Contribuir con la acción transformadora de la educación a partir del proceso de integración escuela-comunidad.

Identificar los elementos necesarios para el logro de la acción transformadora en educación.

Describir los elementos necesarios para el logro de la acción 
transformadora en educación.

Estructurar un esquema representativo que visualice los elementos acción transformadora de la educación a partir del proceso de integración escuela-comunidad.

\subsection{Elementos para la Acción Transformadora a partir de la Participación Ciudadana entre la Escuela y la Comunidad}

La generación de una teoría centrada en la Participación Ciudadana para la Transformación de la escuela se basó en una serie de categorías emergentes, las cuales se describen a continuación:

1.- Participación Ciudadana: Las personas inmersas en esta participación, requieren mantener el respeto al estado de derecho, el acceso a componentes institucionales, a la información y la confianza de los ciudadanos en las instituciones democráticas.

2.- Integración Escuela-Comunidad: Esta integración requiere de alianzas entre la institución educativa y la comunidad, para la realización de trabajo mancomunado en la planificación, organización, ejecución y control de programas o proyectos a ser desarrollados en la escuela o en la comunidad.

3.- Acción Trasformadora: Una escuela, que establece sinergia y relación con la comunidad local, con otras instituciones educativas, con el Consejo Comunal del sector, con los entes gubernamentales establecidos en la comunidad, como Alcaldía, organizaciones populares como los Comités Locales de Abastecimiento y Producción (CLAP), el Instituto Autónomo Consejo Nacional de Derechos del Niño, Niña y Adolescentes (IDENA), entre otros.

4.- Contexto Escolar: Concebido como el ámbito de la escuela y el entorno que la rodea, observándose entre estos factores la localización de la comunidad, el nivel económico de los habitantes, los servicios que poseen en el sector, áreas verdes o recreativas; enfocándose estos en cómo afectan el 
rendimiento académico, emocional y físico de los estudiantes dentro de la escuela.

\section{Hallazgos}

En función de los objetivos específicos y las interrogantes planteadas se puede mencionar, que la participación ciudadana es la relación que existe entre los padres y representantes y el personal del plantel, realizando acciones significativas que ayuden al mejoramiento tanto de la misma escuela como de la comunidad en general.

- Los docentes le dan un significado aparente al accionar de los padres y representantes en el plantel desde un servicio educativo, dejando la responsabilidad a los docentes y directivos de toda la gestión educativa, en este sentido, la participación ciudadana desde la relación escuela-comunidad es escasa, limitándose únicamente a las acciones administrativas del plantel, a saber, asistencia a reuniones, retiro de boletas informativas, reclamos por problemas entre pares, entre otras, lo que hace evidente la poca importancia dada a la acción transformadora de la escuela.

- La participación ciudadana cuenta con elementos como: compromiso, responsabilidad, visión compartida del futuro, igualdad de derechos, colaboración, planificación en conjunto, los docentes, padres y consejos comunales, ya que la escuela no es aislada de la comunidad, sino al contrario, forma parte de ella.

- Las estrategias de participación que en algún momento se utilizaron han dejado de existir pues la Dirección del Plantel es la que "resuelve todo" y no da cabida a los demás para participar.

- Sin embargo, desde la perspectiva comunitaria, los padres y representantes que conforman las vocerías de los diferentes Consejos Comunales que circundan el sector, muestran interés en las actividades externas a la institución escolar y que los beneficia de manera directa como es 
la elaboración de proyectos comunitarios para las mejoras comunitarias, organización y participación en el comité de alimentación, para la adquisición de los alimentos a través del CLAP.

- Solo hay una relación de servicio, la escuela ofrece un beneficio a la comunidad y ya, pero la comunidad no devuelve ese accionar, por lo tanto, se puede señalar que no hay relación para la acción transformadora_del proceso educativo y mucho menos de la comunidad, ya que si a la escuela no se involucra como se avanza.

- Mientras la comunidad no asuma el compromiso y la responsabilidad de la escuela, no hay acción transformadora, para que esto ocurra, debe existir una articulación entre la escuela y la comunidad, una convivencia pacífica en los sectores, intercambio de ideas, que se tome en cuenta al directivo, a los docentes.

\section{Conclusiones}

En función de los hallazgos, la autora de esta investigación concluye que se deben tomar acciones dirigidas a lograr la Acción Transformadora de la Escuela y la Comunidad a través de la implementación de estrategias para el incremento de la participación ciudadana, ya que la misma permitirá la consolidación de equipos de trabajo, colaboración de los padres, madres y representantes en las labores escolares, la satisfacción de las expectativas de la institución educativa y la comunidad, el compromiso con la meta que propicien la acción transformadora de la escuela y la comunidad.

Es fundamental, la implicación de la familia para beneficiar la convivencia y un clima socioafectivo favorable para el libre desarrollo integral de los estudiantes, quienes también son actores para la transformación que requiere la escuela y la comunidad.

En tal sentido, para que la acción transformadora se de en la articulación, es necesario que cada actor educativo asuma un compromiso, 
una responsabilidad con ellos mismos, ya que es un requisito importante en el potencial humano, para dicha transformación.

Para lo anterior, es necesario, que los padres, madres y representantes participen en las actividades que se realizan en la escuela, con espíritu de motivación, interés, con la mayor disposición para el alcance de la integración escuela-comunidad.

\section{Referencias}

Borja, R. (2002-2003). Enciclopedia de la política. Fondo de Cultura Económica, México, 3aㅡ ed. corr. yaum., 2 vols.

Currículo Nacional Bolivariano (2007). Diseño Curricular del sistema Educativo Bolivariano. Ministerio de Educación Superior, Ministerio del Poder Popular para la Educación. Versión impresa ISSN: 13164910. Educere, vol. 11 nro. 39. Mérida, Venezuela.

Fermín, J. (2003). Herramientas de Participación Ciudadana y contraloría Social. Proyecto componente formativo del programa de fortalecimiento técnico de los consejos locales de planificación pública. Ministerio de Planificación y Desarrollo. Caracas: Venezuela.

Martínez, M. (2006a,b,c). La Investigación Cualitativa Etnográfica en Educación. Caracas, Venezuela: Texto.

Mosonyi, E. (2007). El concepto de interculturación. Facultad de Economía y Ciencias Sociales. Universidad Central de Venezuela. Caracas: Venezuela.

Pérez, A. \& Salazar, A. (2010). Modelos de diseños curriculares en la Educación Básica venezolana. Revista de Teoría y Didáctica de las Ciencias Sociales. Versión impresa. ISSN: 1316-9505.

Proyecto Nacional Simón Bolívar, Primer Plan Socialista - PPS (2007-2013).

Desarrollo Económico y Social de la Nación. Caracas: Venezuela

Rey, R. \& Santamaría, J. (2010). La educación en un contrato de calidad. 
Barcelona: Monografías, Escuela española.

Rusque, A. (2003a,b,c). De la Diversidad a la Unidad en la Investigación Cualitativa. Caracas, Venezuela: Vadell Hermanos.

Universidad Pedagógica Experimental Libertador (2002). Manual de Trabajos de Grado de Especialización y Maestrías y Tesis Doctorales. 4ta ed. Caracas, Venezuela: Autor. 


\section{Sonia María Peña Guzmán}

e-mail: profesorasonia12@gmail.com

Nacida en Venezuela. Profesora de Castellano,

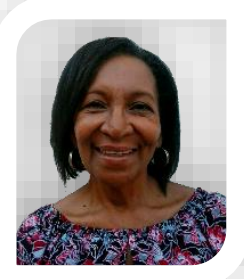
egresada de la Universidad Pedagógica Experimental Libertador "Rafael Alberto Escobar Lara". Especialista en Planificación y Evaluación de la Educación, Universidad Santa María. Magister Scientiarum en Educación Superior, Universidad Nacional experimental de la Fuerza Armada Nacional Bolivariana (UNEFA) Doctorante en Ciencias de la educación, Universidad Latinoamericana y del Caribe (ULAC), Caracas, Venezuela. Profesora de postgrado UNEFA, Miranda, Venezuela. Coordinadora de la carrera Maestría en Educación Superior, UNEFA. Profesora jubilada en el área de Castellano. Directora en ejercicio de la Unidad Educativa Estadal "Dolores González".

El contenido de este manuscrito se difunde bajo una Licencia de Creative Commons ReconocimientoNoComercial-Compartirlgual 4.0 Internacional 